\title{
Research on Optimization of Blockchain Network and Data Communication in the Ecological Structure of Sports Industry
}

\author{
Yupeng Li, ${ }^{1}$ Kitak Kim, ${ }^{2}$ and Yu Ding $\mathbb{D}^{1}$ \\ ${ }^{1}$ College of Physical Education and Health, Linyi University, Linyi, 276000 Shandong, China \\ ${ }^{2}$ Department of Leisure Sports, Pai Chai University, Daejeon 302735, Republic of Korea \\ Correspondence should be addressed to Yu Ding; dingyu@lyu.edu.cn
}

Received 21 June 2021; Revised 29 July 2021; Accepted 27 August 2021; Published 27 September 2021

Academic Editor: Zhihan Lv

Copyright (C) 2021 Yupeng Li et al. This is an open access article distributed under the Creative Commons Attribution License, which permits unrestricted use, distribution, and reproduction in any medium, provided the original work is properly cited.

\begin{abstract}
Problems such as excessive centralization of sports industry operations, unclear value, and lack of IP protection hinder the benign interaction between various formats of the sports industry and the healthy operation of the industrial ecosystem. This article is aimed at studying the use of the decentralization, openness and transparency, smart contracts, copyright traceability, and other characteristics of the BT to construct a PCMR model, which can solve the pain points faced by the sports industry ecosystem in a targeted manner, and forming a way to reconstruct trust, the specific implementation path of the integration of BT and sports industry with mechanism as the core. This paper proposes blockchain network and data communication algorithms such as consensus algorithm, signature core algorithm, and practical Byzantine fault-tolerant algorithm to provide technical support for the application of blockchain technology in the sports industry ecosystem and, at the same time, provide technical support for blockchain network and data communication. The feasibility analysis of the application of the sports industry ecosystem is proposed, and the implementation path of the blockchain network and data communication to reconstruct the sports industry ecosystem is analyzed. Finally, the empirical test of the support of blockchain sports to the sports industry is carried out. By analyzing the panel data of 240 observations of 48 listed companies in 5 years as a sample of the sports industry, the experimental results show that the dominant modulus of the sample does not have other differences, and the leftovers all pass significance test at the $10 \%$ level at least, denoting that the fundamental sample is more rational.
\end{abstract}

\section{Introduction}

1.1. Background. With the development of social economy, the agglomeration effect of industries has become increasingly prominent. The scarcity of resources and the complementarity of links determine that the various elements of the industry also have the characteristics of interdependence, interconnection, mutual restriction, and mutual influence. The "industrial ecosystem" is this. A concept was also put forward-its essence is a structural layout based on the benign operation of various elements of the industry and the effective matching of resources. Any industry with diversified products and initial scale of the industrial chain needs to build an industrial ecosystem naturally or consciously. With the advent of the 5G era and the advancement of "new infrastructure," major countries in the world are accelerating the industrialization of blockchain technology. The key to the concrete landing and commercial application of blockchain technology is to empower the physical industry and promote the industry. However, the ecosystem structure of my country's sports industry is not yet perfect, and the industrial chain and ecosystem network are not yet perfect. The introduction of the basic concepts and technology of $\mathrm{BT}$ in the sports industry can effectively stimulate the internal vitality of industrial elements and promote a virtuous circle of the sports industry ecosystem at the beginning of the industrial chain.

1.2. Significance. Using industrial structure theory to analyze and study industrial development has been one of the hotspots studied by global scholars in recent years, and my country's sports industry is facing constant opportunities and challenges, among which the problem of industrial structure is the bottleneck restricting its development. Grasp 
the composition, connection, and change trend of various elements in the development of the sports industry, find out the problems and shortcomings, put forward countermeasures to optimize the structure of my country's sports industry, and provide part of the theoretical basis for the government to formulate reasonable sports industry development plans and policy measures, to contribute to the realization of our country's progress from a "sports power" to a "sports power." At the same time, the computational efficiency and storage space of the blockchain will improve with the continuous updating of computer hardware configurations, and the improvement of data transmission efficiency requires a reasonable communication model and effective data communication algorithms. The maturity of the two technologies can promote the reform and development of the ecological structure of the sports industry. This article will focus on solving the problem of blockchain data transmission performance optimization and plan the blockchain data transmission path through scientific and reasonable methods. In the face of distributed data communication, it can transmit data quickly, reliably, and fairly, thereby optimizing sports ecological structure, improve work efficiency, and improve user experience.

1.3. Related Work. By measuring health and exercise information, it is possible to quantitatively evaluate the amount of exercise and fitness level of sports athletes, thereby ensuring effective fitness effects. Shan and Mai's research found that the development of BT and Internet of Things technology provides a new perspective and technical means for fitness management technology [1]. In response to the current problems in the field of sports and fitness, he designed and implemented the dynamic management technology of sports and fitness based on the concepts of the Internet of Things and BT. On the basis of in-depth analysis of the domestic and foreign health Internet theory research and application status, theories and methods of sports information and health information collection are studied. Temperature sensor and acceleration sensor are used to collect human body temperature and exercise steps and then estimate human health and exercise conditions. With the rise of Bitcoin and its underlying foundation, the BT, the sports industry has undergone major technological changes in its environment. Naraine's research shows BT and conducts conceptual research in sports and discusses the impact and value proposition of BT on the sports industry. After a brief overview of the BT and technology stack, the mechanism is conceptually rooted in the network paradigm, which is already known in the academic sports world. This approach believes that it is worth noting that the ability of BT to promote new sources of income and improve data management is determined, and it is recommended that sports management and communications consider the value of BT and technology stacks, because the industry's digital footprint continues to increase and become more and more complex [2]. However, his experimental process is not closed, so there is a certain deviation in the experimental results. Since its establishment, BT technology has shown attractive development prospects. From the initial cryptocurrency to the cur- rent smart contract, BT has been used in many fields. Although some research has been conducted on the security and privacy issues of the $\mathrm{BT}$, there is a lack of systematic research on the security of the BT system. Based on several of the most popular BT systems (such as Ethereum, Bitcoin, and Monero), A X L has conducted a systematic investigation of the security threats of the BT and listed relevant actual attack cases. Then, he summarized the security enhancement solutions of the BT and provided guidance for the healthy development of the BT [3]. Although the research perspective is forward-looking, there are still many unachievable parts of the technology.

1.4. Innovation. The innovation of this paper is (1) analyze the development status of my country's BT sports industry ecosystem through market research and data collection. At the same time, it puts forward a feasibility analysis of the application of BT in the sports industry ecosystem and analyzes the implementation path of $\mathrm{BT}$ reconstruction of the sports industry ecosystem, and finally, an empirical test of BT sports supporting the sports industry. (2) Propose BTrelated algorithms such as consensus algorithm, signature core algorithm, and practical Byzantine fault-tolerant algorithm to provide technical support for the application of BT technology in the sports industry ecosystem.

\section{BT-Related Algorithms}

MEC is the key technology and architecture of 5G communication evolution. It represents the transition from a mobile broadband network to a software-defined network. Figure 1 shows a simplified version of the MEC architecture, where the MEC server provides edge computing capabilities for terminal devices.

With the development of blockchain networks and data communications, different perception network architectures have emerged according to different application scenarios. This article presents a blockchain network and data communication system architecture. As shown in Figure 2, using the data transmission process, the blockchain network and data communication are divided into three layers, namely, the mobile sensing layer, the transmission layer, and the service processing application layer. On the basis of this architecture, it can be extended according to different scenarios. For example, a privacy protection layer can be added to the mobile perception layer. All data perceived by the user is not directly uploaded to the service platform but sent to the server after processing on the mobile smart end. In order to improve the operating efficiency of the system and ensure the real-time performance of the data, a special data channel layer can be added to the transmission layer to give priority to the rapid transmission of special data to the server and so on.

2.1. Consensus Algorithm. The distributed consensus problem originated from the classic Byzantine generals problem proposed by Leslie Lamport in 1982. Lamport proved that when the proportion of malicious nodes does not exceed $1 / 3$, there is an effective algorithm to keep honest nodes 


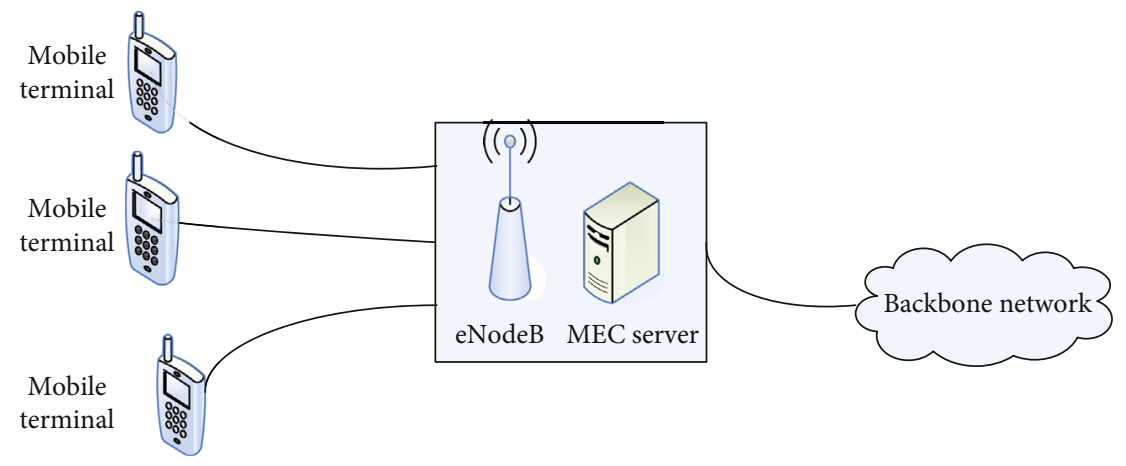

FIGURE 1: MEC architecture.

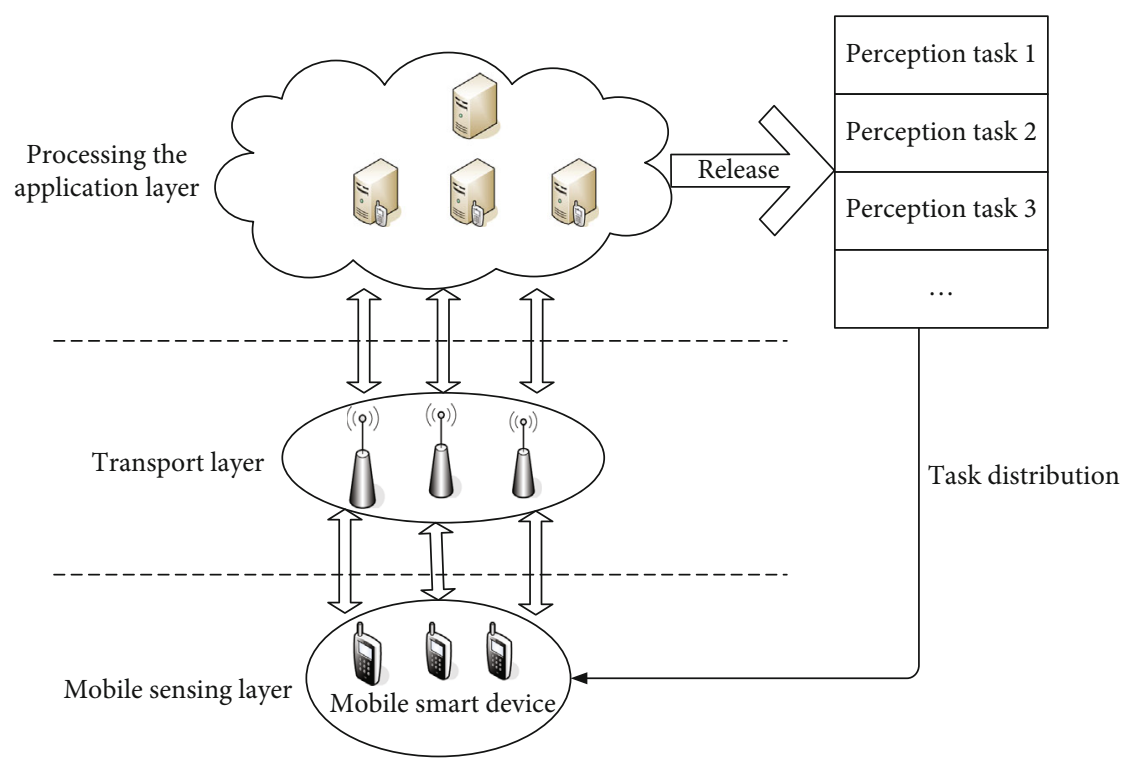

FiguRE 2: Blockchain network and data communication three-tier architecture model.

consistent $[4,5]$. How to ensure the consistency of honest node data is the core problem of the BT. In order to solve this problem, the industry and academia have provided a series of solutions [6].

2.1.1. Proof of Work Algorithm. In Bitcoin, the Proof of Work (POW) algorithm was creatively introduced to solve the distributed consensus problem in the BT $[7,8]$. The core idea is to find a suitable Nonce value as an input parameter of the algorithm interface. After calculation, the result is lower than the specific difficulty threshold. The principle is shown in

$$
\operatorname{Hash}(h, r) \leq \frac{M}{d}
$$

In the formula, $h$ represents the hash value of the block header of the packed block, $r$ represents a random number, $M$ represents the maximum value of uint256, and $d$ represents the difficulty coefficient [9]. The node increments the Nonce until it finds a suitable random number [10]. The fastest completed node obtains the accounting right of the block, packs the block, and broadcasts it to the entire network.

2.1.2. Proof of Equity Algorithm. The POW algorithm needs to consume a lot of computing power for "mining," and this part of the computing power has not received actual benefits $[11,12]$. Therefore, a consensus algorithm based on Proof of Stake (POS) is proposed, which competes for the right to bookkeeping through the number of coins held and the holding time. The core idea is to calculate the coin age, as shown in

$$
C_{a}=\sum_{i=0}^{n} \text { age }_{i}
$$

2.2. Signature Core Algorithm. In the BT system, the signature algorithm is mainly used to verify the legitimacy of the transaction and the identity information of the sender of the transaction [13]. In addition, the signature algorithm can protect the privacy of transaction information, including 
the address information of both parties to the transaction, transaction amount, etc. $[14,15]$. In this scheme, the transaction amount of transactions involving multiple input addresses and multiple output addresses can be hidden. Without loss of generality, the transaction is divided into input and output.

A transaction consists of $n$ inputs and $m$ outputs and has $\sum_{i=1}^{n} \mathrm{in}_{i}=\sum_{j=1}^{m}$ out $_{j}$. For any $i$ and $j, 1 \leq i \leq n, 1 \leq j \leq m$, in order to hide in and out, this paper uses an elliptic curve algorithm for protection [16]. Choose $G$ as the generator of $\mathrm{Fp}$, and the transaction forms of in and out $_{j}$ are $I_{j}=\mathrm{in}_{j} \cdot G$ and $O_{j}=\mathrm{Out}_{j} \cdot G$. According to the calculation rules of the elliptic curve, we can get

$$
\begin{aligned}
\sum_{i=1}^{n} \mathrm{in}_{i} \cdot G & =\sum_{i=1}^{n} I_{i}=\left(\sum_{i=1}^{n} \mathrm{in}_{i}\right) \cdot G, \\
\sum_{j=1}^{m} \mathrm{Out}_{j} \cdot G & =\sum_{j=1}^{m} O_{j}=\left(\sum_{j=1}^{m} \mathrm{Out}_{j}\right) \cdot G .
\end{aligned}
$$

Through equations (1) and (2), we have $\sum_{i=1}^{n} I_{i}=\sum_{j=1}^{m} O_{j}$, and we can verify $\sum_{i=1}^{n} \mathrm{in}_{i}=\sum_{j=1}^{m}$ out $_{j}$, because the attacker cannot get in ${ }_{i}$ and out ${ }_{j}$ through $I_{i}$ and $O_{j}$, so the transaction amount in this scheme can be hidden $[17,18]$. The homomorphic characteristics and defects of this signature scheme are given below.

The homomorphism proof of the signature scheme, considering the development of quantum computing, homomorphism is an important characteristic for evaluating the security of an algorithm [19]. The additive homomorphism of the basic scheme given in equation (1) can be proved. Proof: for each $i, 1 \leq i \leq n$, through the algorithm of an elliptic curve, we can get

$$
\begin{aligned}
\sum_{i=1}^{n} \mathrm{in}_{i} \cdot G & =\left(\sum_{i=1}^{n} \mathrm{in}_{i}\right) \cdot G, \\
\left(\sum_{i=1}^{n} \mathrm{in}_{i}\right) \cdot G & =\sum_{i=1}^{n} \operatorname{in}_{i} \cdot G .
\end{aligned}
$$

Further, you can get

$$
\left(\sum_{i=1}^{n} \operatorname{in}_{i}\right) \cdot G=\sum_{i=1}^{n}\left(\operatorname{in}_{i} \cdot G\right)
$$

The left side of equation (4) indicates that the addition operation is performed first and then the encryption operation is performed, and the right side of the equation indicates that the encryption operation is performed first and then the addition operation is performed [20,21]. So it can be proved that the basic signature scheme has additive homomorphism.

The flaws of the basic signature scheme, the basic signature scheme can hide the transaction amount of a transaction containing multiple input addresses and output addresses, but the attacker can still obtain the transaction amount by some means. For the given basic signature algorithm, assuming that the malicious attacker controls $u$ inputs and $v$ outputs, it is satisfied that $\sum_{i=1}^{u} \mathrm{in}_{i}=\sum_{j=1}^{v}$ out $_{j}$, in the normal network; the sum of all input amounts is

$$
\text { Is }=\sum_{i=1}^{n} \operatorname{in}_{i}-\sum_{j=1}^{u} \text { int }_{j} \text {. }
$$

The sum of all output amounts is

$$
\text { Os }=\sum_{i=1}^{n} \text { out }_{j}-\sum_{j=1}^{u} \text { out }_{j} .
$$

The set $\left\{\text { int }_{j}\right\}_{1 \leq j \leq u}$ and $\left\{\text { out }_{j}\right\}_{1 \leq j \leq v}$ are the set $\left\{\text { int }_{i}\right\}_{1 \leq i \leq u}$, a subset of $\left\{\text { out }_{i}\right\}_{1 \leq i \leq u}$. Because it is easy to get $\sum_{i=1}^{u} \mathrm{in}_{i}=\sum_{j=1}^{v}$ out $_{j}$ and $\sum_{i=1}^{n} \mathrm{in}_{i}=\sum_{j=1}^{m}$ out $_{j}$, we can get Is $=$ Os, so we can verify Is $\cdot G=$ Os $\cdot G$. Therefore, if the verification fails, the attacker cannot succeed in the attack.

2.3. Practical Byzantine Fault-Tolerant Algorithm. The Practical Byzantine Tolerance Algorithm (PBFT) solves the problem of confusion in the Byzantine algorithm by introducing a master node. Its high transaction efficiency and performance, high availability, understanding, and other benefits make it a recommended distributed consensus algorithm in the alliance chain $[22,23]$. The entire process of the practical Byzantine fault-tolerant algorithm is shown in Figure 3.

As shown in Figure 3, node 0 is the master node. In the practical Byzantine fault-tolerant algorithm, only the master node accepts client transaction requests. Through three global broadcast processes, all normal nodes in the entire network reach an agreement. The client receives the same result for more than $1 / 3$ participating nodes of the request, which indicates that the consensus is reached [24].

It is assumed that the node belongs to group $A$, then the public key ring $R_{A}=\left(p k_{1}, p k_{2}, \cdots, p k_{n}\right)$, and the message to be encrypted is $m$, the private key is $x_{i}$, the public key is $p$ $k_{i}=x_{i} \mathrm{G}$, and the public key $p k_{i}$ are obtained by the elliptic encryption algorithm. The process of associating ring signatures is as follows.

Define two mapping functions, $H_{1}:\{0,1\}^{*} \rightarrow Z_{q}, H_{2}$ : $\{0,1\}^{*} \rightarrow G$, and calculate the public key image $y^{\prime}$ as

$$
y^{\prime}=x_{i} H_{2}\left(R_{A}\right) .
$$

Generate random number $\delta \in Z_{q}$, and calculate $c_{i+1}$ according to

$$
c_{i+1}=H_{1}\left(R_{A}, y^{\prime}, m, \partial G, \partial y^{\prime}\right) .
$$

For $j=i+1, \cdots, n, 1, \cdots, i-1$, generate a random number $s_{i} \in Z_{q}$, and calculate $c_{j+1}$ according to

$$
c_{j+1}=H_{1}\left(R_{A}, y^{\prime}, m, s_{j} \mathrm{G}+c_{j} \mathrm{p} k_{j}, s_{j} H_{2}\left(R_{A}\right)+c_{j} y^{\prime}\right) .
$$




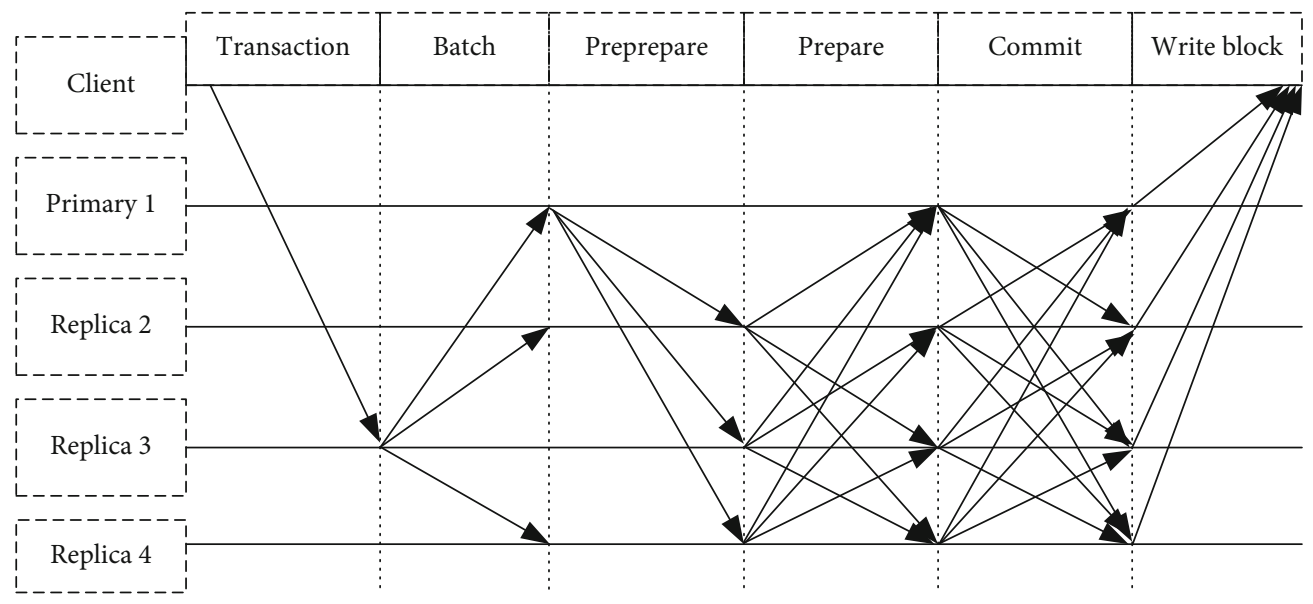

Figure 3: Practical Byzantine fault-tolerant algorithm process.

Calculate $s_{i}$ according to

$$
s_{i}=\partial-x_{i} c_{i} \bmod q .
$$

Generate signature $\delta_{R G}(m)$ according to

$$
\delta_{R G}(m)=\left(c_{1}, s_{1}, \cdots, s_{n}, y^{\prime}\right)
$$

After the ring signature is processed, the node broadcasts the PREPARE message to the primary domain node: $<<$ PREPARE, $v, n, d, G>, \delta_{R G}(m), R_{G}>$, where $G$ represents the group, if the node belongs to the $A$ group, then $G=A, R_{G}$ represents the public key ring of the members of the $G$ group, and $\delta_{R G}(m)$ represents the ring signature of the message by the node.

\section{BT and Sports Industry Ecosystem Integration}

The sports industry takes the IP created by sports activities and sports events as the core and takes the authorization of sports IP as the extension of the industry. The business types involved are very rich. Therefore, a reasonable sports industry ecosystem consists of many industrial elements at the core, peripheral, and related layers. The structured system is shown in Figure 2.

It can be seen from Figure 4 that the core layer of the sports industry ecosystem is the eight subindustries that are most closely related to sports IP, that is, specific sports events, and it contains almost all the content of the sports industry in the narrow sense. The sports products consumed by the masses mainly come from the core layer. The core layer not only provides tangible material products such as sports goods, sports lottery, and sports venues but also provides intangible service products such as competition performances, fitness training, and celebrity brokers. Regardless of the motivation, the core of the sports industry is sports and its experience, and other industries at the core are closely focused on sports.
3.1. Fit of BT and Sports Industry Ecosystem. In the technical framework of the BT, not only is every transaction open and transparent, the value of the items for trading can be quantified, and the ownership and whereabouts of the items will also be publicly recorded. Traders on the chain must abide by the rules, the cost of violations will be greatly increased, and when all transactions are open, the value is determined, and the copyright attribution is clear; piracy and infringement will be avoided to the greatest extent; even if it occurs, the accountability process is also clear.

Another important feature of the BT is the automatic generation of smart contracts. That is, when both parties to the transaction meet the transaction conditions and there is no violation in the transaction process, the agreement automatically takes effect. BT transactions are carried out by both parties in a completely unfamiliar and without a central organization. The content of the smart contract includes the specific provisions of the transaction and the respective obligations and rights of both parties. The binding force of smart contracts on transaction behavior is embodied in the following: if one party violates the rules of the contract immediately terminates, only when all parties meet the transaction requirements can the transaction result be produced. The transaction information of the two parties is written into the digital contract, and the transaction process is subject to fair digitization. The BT reshapes the trust mechanism of the transaction, maximizes the credibility of the transaction, and ensures the smooth progress of the transaction. The technical characteristics of the BT are shown in Figure 5.

It can be seen from Figure 3 that the decentralization of the BT, the determination of commodity value, the clear attribution of copyright, the immutability of information, the transparency and openness of transactions, the smartness of contracts, etc., are very clearly corresponding to the pain points of the sports industry ecosystem. In addition, the BT can be divided into three forms: public chain, private chain, and alliance chain, and can be flexibly combined according to the different needs of the target object for open features. Therefore, the technical logic of the BT provides for the reconstruction of the sports industry ecosystem basis. 


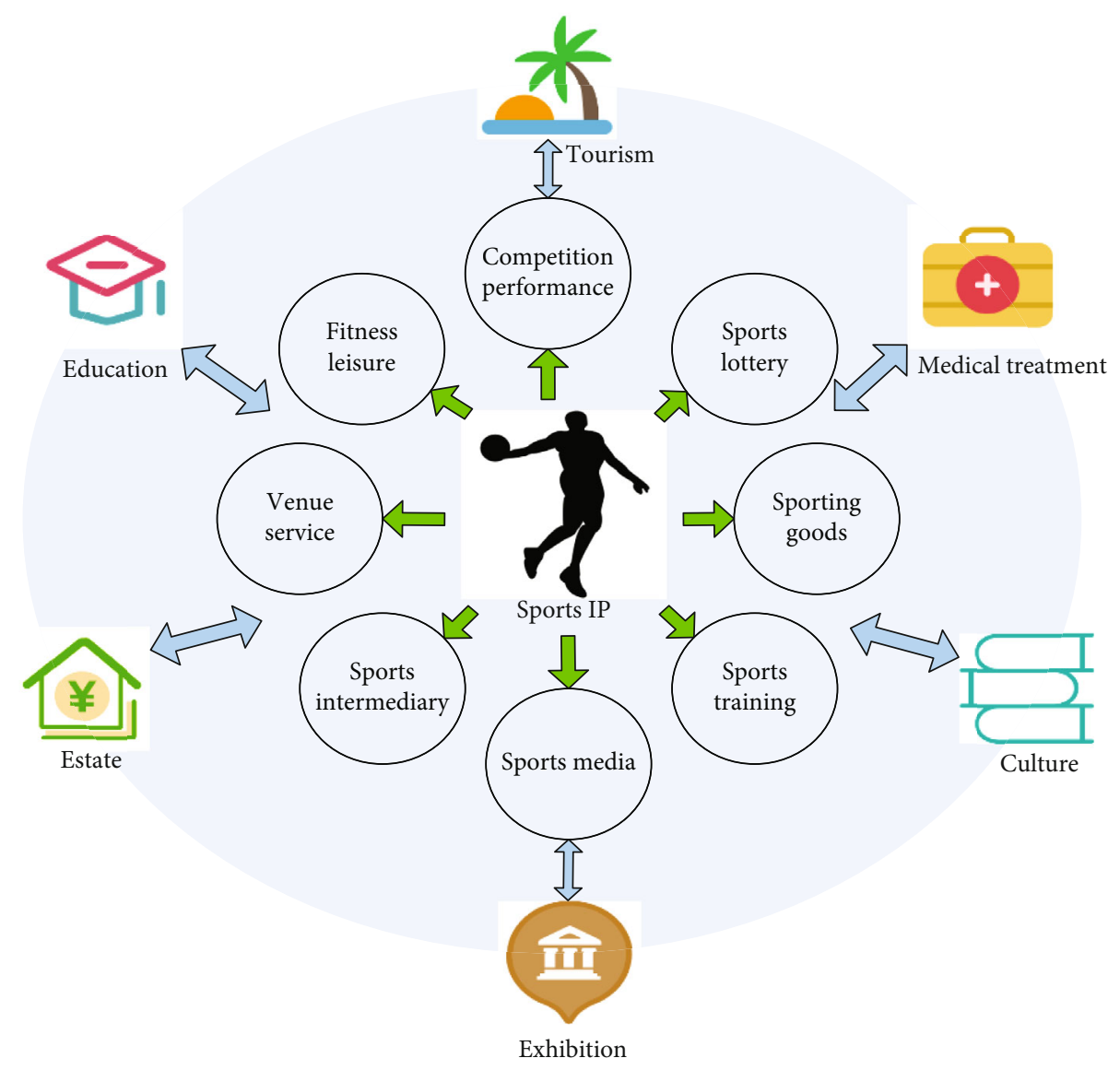

FIgURE 4: The basic structure of the sports industry ecosystem.

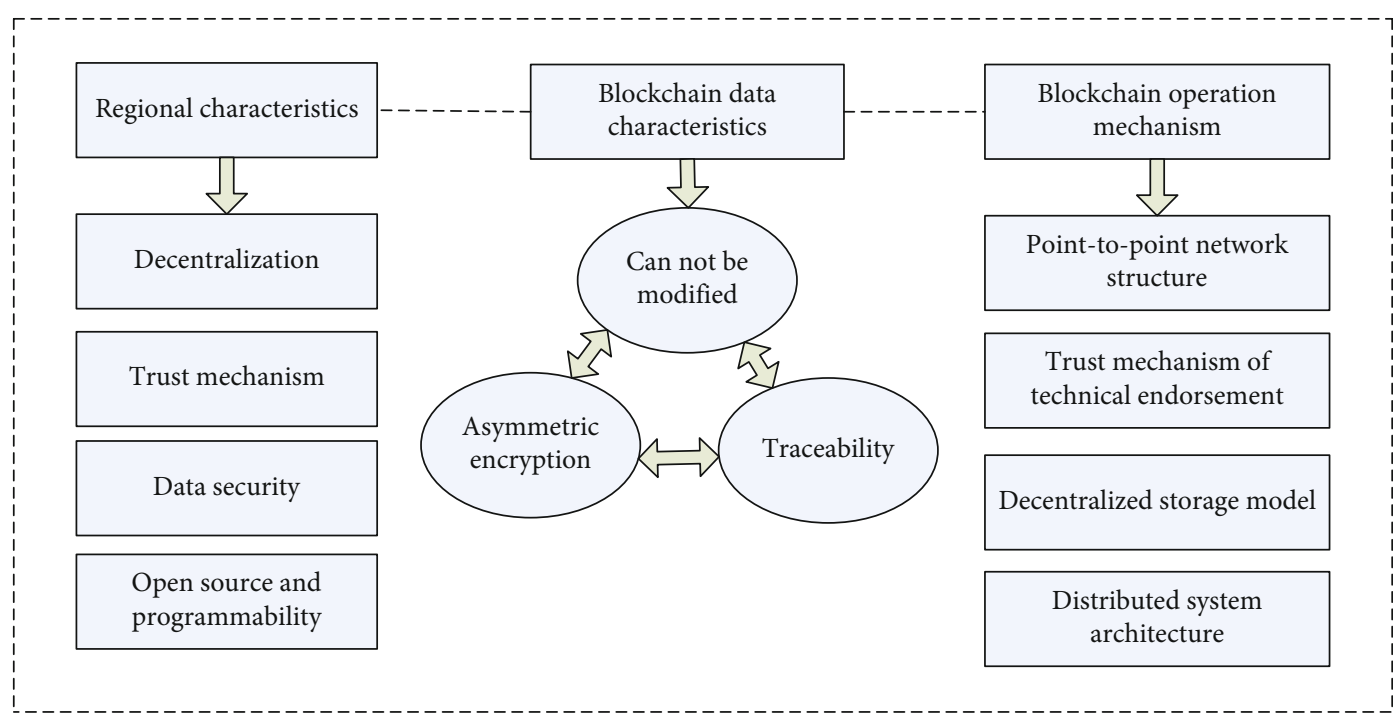

FIgURE 5: BT technical characteristics.

3.2. Implementation Path of Reconstructing the Sports Industry Ecosystem Based on BT. In terms of concepts and technologies, BT can be applied to different levels of the core, peripheral, and related layers of the sports industry ecosystem. Therefore, in combination with the more prominent pain points in the development of the sports industry ecosystem, follow specific issues $\rightarrow$ corresponding feature$s \rightarrow$ solutions $\rightarrow$ the logical idea of the final result. The construction of a PCMR theoretical model integrating the BT and the sports industry is conducive to hierarchically designed solutions to the problems of sports industry operations. 
3.2.1. Solve the Imbalance of Industrial Proportions. The output value of my country's sports industry is mainly concentrated in the low-end sports goods manufacturing field. Compared with countries with developed sports industries, it has fewer high-end sports brands and sports technology patents. At the same time, in the core competition field, the intellectual property resources of my country's top core competitions are relatively scarce, which cannot fully meet the needs of the audience. Figure 6 shows the number and proportion of application distribution fields of my country's BT enterprises.

It can be seen from Figure 4 that although the application of BT technology is becoming increasingly diversified, it is less used in the field of sports. The decentralized confirmation value and smart contracts of the BT can coordinate the industry's supply and demand ratio. An exclusive private chain can be established in a sports enterprise for data management and auditing within the company, enabling efficient management within the company. In the production process, the company can use the data information obtained in the public chain and use big data, artificial intelligence, and other front-end technologies to further evaluate the production design, release channels, and scale of sports products, which can not only meet the market demand to the greatest extent. It can also reduce the risk of overcapacity caused by insufficient market information.

3.2.2. Stimulate the Vitality of the Enterprise. The main reasons for the lack of vitality of current sports enterprises are the insufficient market development, fierce competition in the homogenization of the industry, and the lack of public consumption enthusiasm. Insufficient marketization has led to unclear parts of the industry, lack of openness has led to resource tilt and homogenized competition, and mass consumption has not been thoroughly explored and has restricted the development space of the sports industry. Table 1 shows the changes in the scale of my country's sports industry and the BT industry.

It can be seen from Table 1 that the scale of the BT industry has increased from 130 million yuan in 2016 to 1 billion yuan in 2018, both of which have grown rapidly and have huge market potential. Based on the market logic, if you want to apply blockchain technology in the sports industry, you must rely on market entities and put people's needs as the center. From the perspective of the blockchain market, the global spending in 2018 and 2019 is approximately US $\$ 1.5$ billion and US $\$ 2.9$ billion, respectively, and is expected to reach US\$12.4 billion in 2022; on the one hand, this rapid growth reflects to a certain extent that consumers have a relatively strong demand for the sports industry and the BT market. On the other hand, the integration of sports industry and BT technology can stimulate corporate creativity and reconstruct supply methods in an open, transparent, and standardized transaction process. Therefore, a public chain of the sports industry can be constructed, and a high degree of decentralization can be achieved in a model with a BT as the bottom frame. Since the public chain is completely open and transparent, everyone can participate in the construction of information in the chain and can accommodate a larger audience, but the efficiency of transactions in the chain is relatively low. Therefore, the main purpose of the sports industry public chain is to share the internal information of the sports industry. Collect customers' consumption preferences.

3.2.3. Clarify the Relationship between Government and Enterprise Responsibilities. The nonseparation of powers and responsibilities between government and enterprise not only hinders the further division of labor in sports enterprises but also causes overlapping management agencies and redundant review procedures, which affects the efficiency of sports industry operations and restricts the penetration of the sports industry ecosystem. The decentralization of the BT can help companies and regulators to clarify their respective division of labor. Its open and transparent transaction process and smart contracts can minimize the cost of seeking third-party regulators and realize sports industry operators and regulators.

3.2.4. Avoid Fraud. Falsifications such as match-fixing and false ticketing have seriously dampened the public's trust in the sports industry and hindered its healthy development. The root cause of the false phenomenon is the opacity of the industry and the irregular transaction process. Due to excessive centralization, sports industry transactions have a certain degree of monopoly and closedness. Not only the public cannot supervise but also other internal organizations such as enterprises and industry associations cannot exercise their supervisory powers. The closed nature of transactions leads to a vicious circle of supervision that cannot be carried out normally, trust mechanisms are difficult to establish, infringements occur, and public trust is lost. In the BT, every transaction is open and transparent and recorded by everyone on the chain, so it also has the characteristic of not being tampered with.

\section{Empirical Test of BT Sports Supporting the Sports Industry}

\subsection{Sample Selection and Variable Definition}

4.1.1. Sample Selection. Since my country's BT sports started in 2013 and there are no specific data statistics for the sports industry in practice, there is little data accumulation. Taking into account the above problems, in order to avoid too few empirical samples, the empirical conclusions are not objective and representative. Select the same period of the sports listed companies, and at the same time, eliminate the discontinuous data in the research period, ST or PT listed companies, and finally, get 48 listed companies 5 years with a total of 240 observations of panel data as the sports industry sample. The main variable symbols and definitions are shown in Table 2.

4.2. Model Construction. The cash-cash flow sensitivity model is used to test whether BT sports can provide effective financing support for the sports industry. The basic idea of the cash flow sensitivity model is that the company has a certain cash holding cost, and the company needs to reasonably 


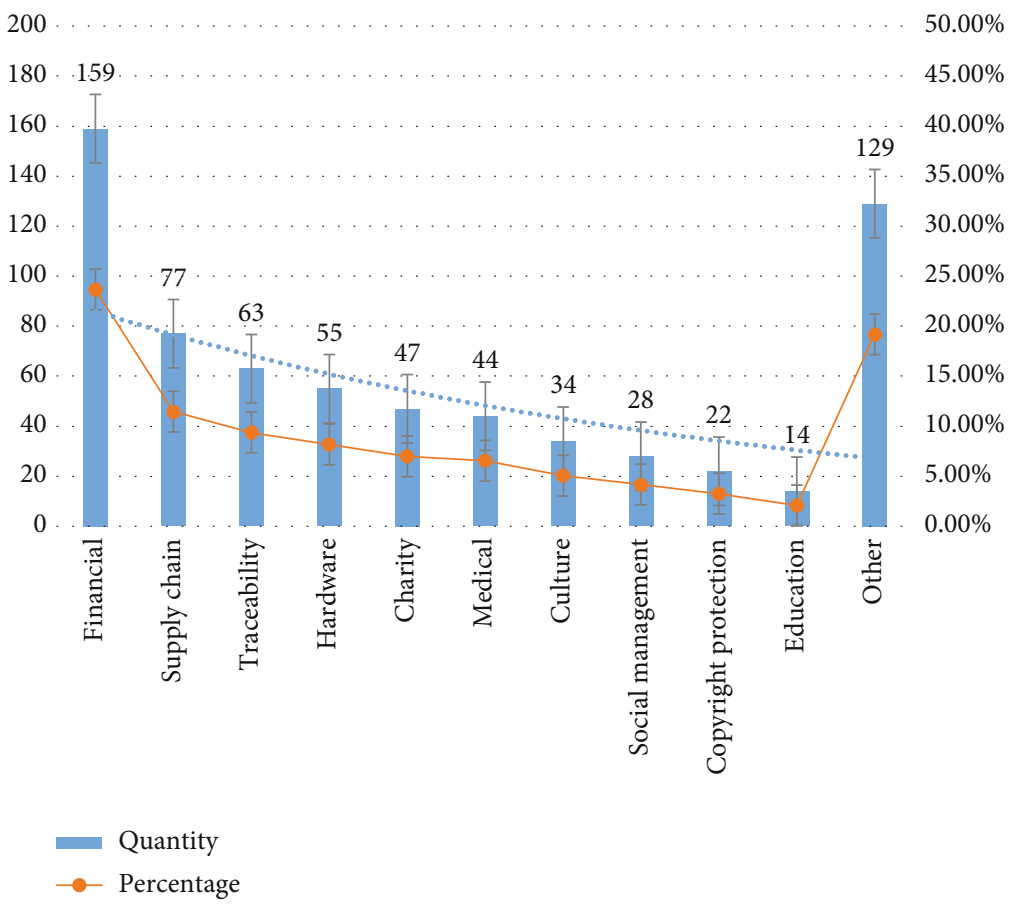

FIGURE 6: The number and proportion of my country's BT enterprise application distribution fields.

TABLE 1: 2015-2018 my country's sports industry and BT industry scale changes.

\begin{tabular}{lcccc}
\hline Year & 2015 & 2016 & 2017 & 2018 \\
\hline Sports industry (ten thousand yuan) & 17107.0 & 19011.3 & 21987.7 & 26579.0 \\
BT industry (ten thousand yuan) & $/$ & 1.3 & 2.4 & 10.0 \\
\hline
\end{tabular}

TABLe 2: Delimit of samples and dominant parameters.

\begin{tabular}{lcc}
\hline Changeable unit and characteristics & & Changeable delimit \\
\hline Explained changeable & $Y$ & Changes in corporate cash and cash equivalents/all corporate property \\
Explanatory differences & $X 1$ & Alter in web cash flow from business activities/total corporate assets \\
& $C 1$ & The product of BT sports scale $(F)$ and $X 1$ \\
Control variable & $C 2$ & The natural logarithm of the company's total property \\
& $C 3$ & All integrated property \\
& $C 4$ & Changes in cashless net working capital/all integrated property \\
& $C 5$ & The velocity of the main business income of the enterprise \\
\end{tabular}

determine its cash holdings based on its financial strength and long-term investment needs. This model uses related measurement methods to check whether the return rate of the cash flow change index generated by the business activities of the cash flow index and the company's equivalent is statistically positive. This shows that the company has a positive impact on cash flow. The response is more sensitive, and there are funding constraints. Otherwise, it does not exist.

Based on this, we will create an empirical model for this article. The explanatory variable of the model is the change of sports company's cash and cash equivalents $(Y)$, and the main explanatory variable is the change of cash flow (X1) generated by the sports company's daily activities. In a similar study, learn how to adjust the multiplication term. BT sports can effectively solve the financial restrictions. At the same time, it references the control variable settings of similar research and subscribes to the related control variable group (C1C5).

That is, $\beta_{1}$, is significantly active; it denotes that my country's sports industry has financing constraints; if the BT sports and generated by the commerce operations are 


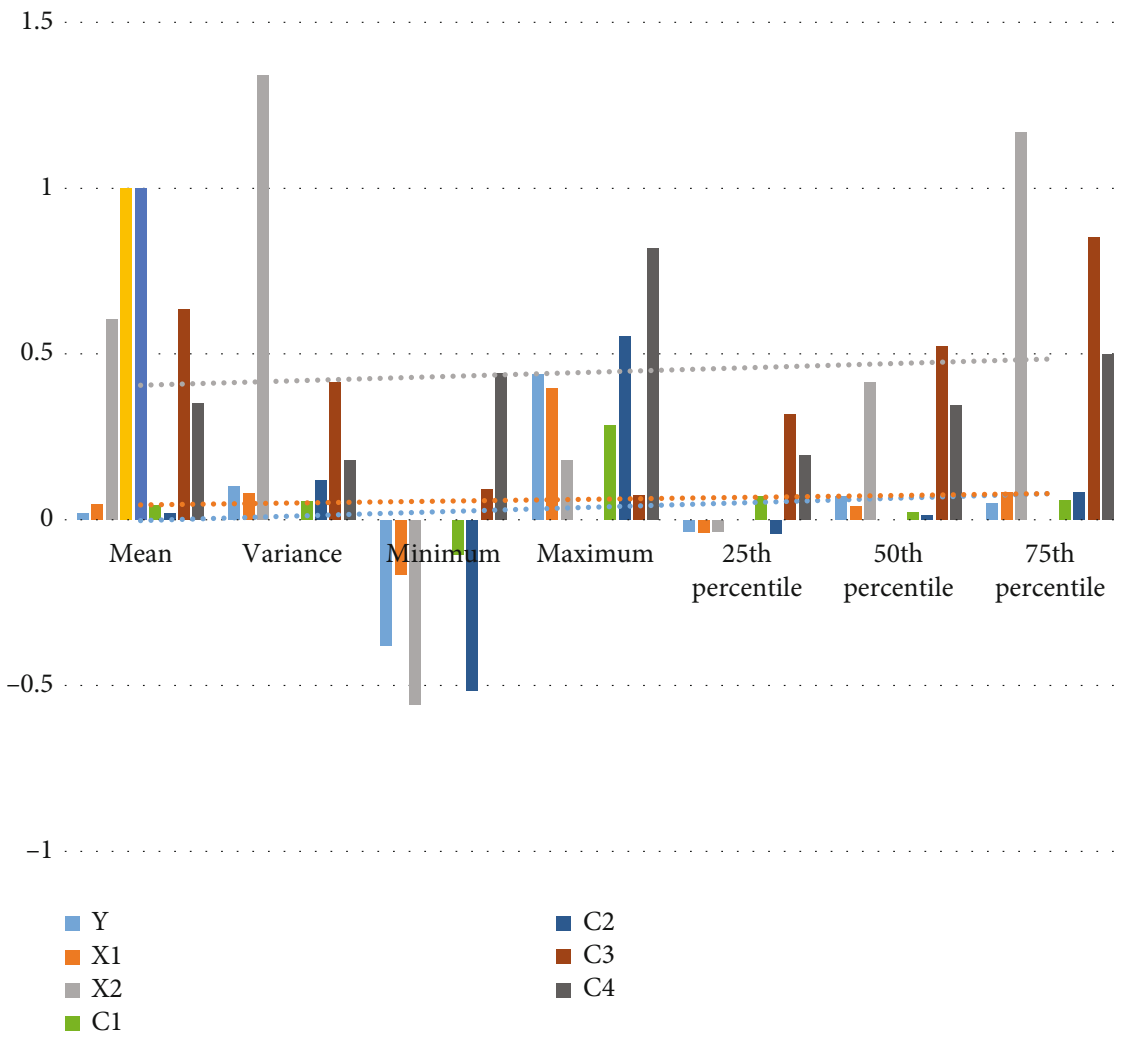

FIgURE 7: Data analysis of dominant parameters.

improved, the data is $\beta$, which is pessimistic. The explanation of related differences in the module is depicted in Table 2. Sample (14) is constructed in the following:

$$
\begin{aligned}
Y_{i t}= & \alpha_{0}+\beta_{1} X 1_{i t}+\beta_{2} X 2_{i t}+\gamma_{1} C 1_{i t}+\gamma_{2} C 2_{i t} \\
& +\gamma_{3} C 3_{i t}+\gamma_{4} C 4_{i t}+\gamma_{5} C 5_{i t}+\varepsilon_{i t},
\end{aligned}
$$

where $i$ represents the $i^{\text {th }}$ company, $t$ represents the $t^{\text {th }}$ year, $\varepsilon_{i t}$ is the random error term, and $\alpha_{0}, \beta_{1}, \beta_{2}, \gamma_{1}-\gamma_{5}$ is the coefficient to be estimated.

Representing the model in matrix form (14) is simplified to

$$
Y_{i t}=\alpha_{0}+\beta_{1} X 1_{i t}+\beta_{2} X 2_{i t}+\theta^{\prime} \cdot C+\varepsilon_{i t},
$$

where $i$ represents the $i^{\text {th }}$ company, $t$ represents the $t^{\text {th }}$ year, $\varepsilon_{i t}$ is the random error term, $\alpha_{0}, \beta_{1}, \beta_{2}$ is the coefficient to be estimated, $\theta^{\prime}$ is the matrix of coefficients to be estimated, and $C$ is the matrix of control variables.

\subsection{Experimental Procedure and Experimental Results}

4.3.1. Data Analysis of Dominant Parameters. Data analysis of dominant parameters is depicted in Figure 7.

As shown in Figure 7, this average change of money and money equivalents $(Y)$ of physical education companies is 0.018 , the change is 0.102 , the lower data is -0379 , and the higher data is 0.439 . The whole difference is huge. The average value of the $X 1$ activities is 0.045 , and the lower data value is -0.167 , denoting that many companies have bad conditions and few money drifts from operating activities. The even parameter (IF) of my country's BT movement is 14,782 , and the fluctuation is 8,111 . Judging from the $20^{\text {th }}$, $50^{\text {th }}$, and $75^{\text {th }}$ percentiles, the scale of my country's sports BT is growing quickly. The even worth of all property $(C 1)$ of the sports company is 21,865 , with a fluctuation of 1,456 , which is a very small range. Judging from the percentages of $20 \%, 50 \%$, and $75 \%$, the difference in the scale of assets of Chinese sports companies is relatively small. The average growth rate $(C 4)$ of major companies in the sports industry was 0.634 , and the fluctuation range was 0.414 . From the lowest price of 20 cents, 50 cents, and 75 cents to the highest price, my country's sports companies have certain income capabilities.

4.3.2. Coefficient Estimation Model Selection. The more commonly used coefficient estimation models for panel data are random-effects model and fixed-effects model. The method to test whether there is a fixed effect or a random effect between variables is the Husman test. The Husman test method is used to test the sample data selected in this paper. The test results, as shown in Table 3, reject the null hypothesis that there is no individual effect at the $1 \%$ significance level, so the fixed-effects model is selected.

4.3.3. Heteroscedasticity Test. Heteroskedasticity means that the sample data does not meet the homoscedasticity assumption of linear regression, and the estimated result obtained at this time is biased. Because this article uses the 
TABLE 3: Husman test results.

\begin{tabular}{lc}
\hline Test the null hypothesis & H0: no individual effect \\
\hline Test result & chi2 $(6)=24.13, P>\operatorname{chi} 2(6)=0.0005$ \\
\hline
\end{tabular}

TABLE 4: Test for heteroscedasticity.

\begin{tabular}{lc}
\hline $\begin{array}{l}\text { Test the null } \\
\text { hypothesis }\end{array}$ & H0: no individual effect \\
\hline Test result & $\operatorname{chi} 2(48)=17569.69, P>\operatorname{chi} 2(48) \leq 0.001$ \\
\hline
\end{tabular}

panel data of listed sports companies, there may be heteroscedasticity between different sections, so the modified Wald test method suitable for panel data heteroscedasticity test is used to test whether this problem exists. The test results are shown in Table 4, indicating that there is heteroscedasticity.

To this end, two methods are used to solve the heteroscedasticity problem: one is the more commonly used white heteroscedasticity correction method; the other is the Bootstrap method. Bootstrap does not make assumptions about the distribution of the data but simulates the overall data through sample data sampling. In this way, the overall distribution is obtained, and the corresponding estimated coefficient significance test is done based on this distribution.

4.3.4. Analysis of Empirical Results. The coefficient estimation outcome of the integrated effect model, respectively, reports the coefficient estimation results under the assumption of homoscedasticity and the white estimation formula to obtain the robust standard error method when heteroscedasticity exists, and the coefficient estimation results obtained by selfsampling 1000 times based on the Bootstrap method. Specific outcomes are shown in Table 5.

Table 5 denotes the experimental outcomes of the mended effect sample. The leftovers are all passing the significance test at the lowest $10 \%$ level that indicates that the fundamental sample setting is rational.

Through the case analysis of the embryonic BT and sports industry integration, we can see that the PCMR model can effectively explain the specific approach of BT integration with the sports industry ecosystem and has a strong explanatory power. Practice has also proved that the basic principles and characteristics of the BT are in line with the elements required in the construction of the sports industry ecosystem. The technology and thinking of the BT can make up for the loopholes and deficiencies in the sports ecosystem in a targeted manner. From a micro perspective, BT technology can realize the business reorganization of the sports industry and solve many pain points in the sports industry. From a macro perspective, using the concept and technology of BT as a framework can solve many problems in the sports industry ecosystem, promote the reconstruction of the sports industry ecosystem, and ensure its virtuous circle. It can be seen that the blockchain can indeed bring huge changes to the sports industry, and practice has also proved that the blockchain has a strong potential to empower the
TABLE 5: Coefficient mended effect sample estimation outcomes.

\begin{tabular}{|c|c|c|c|}
\hline \multirow[b]{2}{*}{ Variable } & \multirow[b]{2}{*}{$\begin{array}{l}\text { Homogeneity } \\
\text { of variance }\end{array}$} & \multicolumn{2}{|c|}{ Heteroscedasticity } \\
\hline & & $\begin{array}{l}\text { Error estimation } \\
\text { outcomes }\end{array}$ & $\begin{array}{l}\text { Bootstrap } 1000 \\
\text { results }\end{array}$ \\
\hline$X 1$ & $\begin{array}{l}0.561 * * * \\
(0.20)\end{array}$ & $\begin{array}{l}0.561 * * * \\
(0.19)\end{array}$ & $\begin{array}{l}0.561 * * * \\
(0.20)\end{array}$ \\
\hline$X 2$ & $\begin{array}{l}0.053 * * * \\
\quad(0.01)\end{array}$ & $\begin{array}{c}-0.023 * \\
(0.01)\end{array}$ & $\begin{array}{c}-0.023 * \\
(0.01)\end{array}$ \\
\hline$C 1$ & $\begin{array}{c}-0.203 * * * \\
\quad(0.06)\end{array}$ & $\begin{array}{l}0.053 * * * \\
\quad(0.01)\end{array}$ & $\begin{array}{l}0.053 * * * \\
\quad(0.01)\end{array}$ \\
\hline$C 2$ & $\begin{array}{c}-0.023 * * \\
\quad(0.01)\end{array}$ & $\begin{array}{c}-0.630 * * * \\
(0.22)\end{array}$ & $\begin{array}{c}-0.630 * * * \\
\quad(0.23)\end{array}$ \\
\hline$C 3$ & $\begin{array}{c}-0.630 * * * \\
(0.20)\end{array}$ & $\begin{array}{c}-0.203 * * \\
\quad(0.08)\end{array}$ & $\begin{array}{c}-0.203 * * \\
\quad(0.08)\end{array}$ \\
\hline$C 4$ & $\begin{array}{l}0.0002 \\
(0.04)\end{array}$ & $\begin{array}{l}0.0002 \\
(0.03)\end{array}$ & $\begin{array}{l}0.0002 \\
(0.04)\end{array}$ \\
\hline C5 & $\begin{array}{l}-0.269 * * * \\
\quad(0.08)\end{array}$ & $\begin{array}{c}-0.269 * * * \\
\quad(0.08)\end{array}$ & $\begin{array}{c}-0.269 * * * \\
(0.09)\end{array}$ \\
\hline$\alpha 0$ & $\begin{array}{c}-1.039 * * * \\
(0.33)\end{array}$ & $\begin{array}{c}-1.039 * * * \\
(0.29)\end{array}$ & $\begin{array}{c}-1.039 * * * \\
(0.34)\end{array}$ \\
\hline$N$ & 240 & 240 & 240 \\
\hline$R^{2}$ & 0.188 & 0.188 & 0.188 \\
\hline $\mathrm{F} / \mathrm{Chi}^{2}$ & 6.131 & 11.278 & 55.41 \\
\hline
\end{tabular}

sports industry, but the financing risks and technical crimes that exist in the application of the blockchain still need to be vigilant and circumvention.

\section{Conclusions}

The integration of BT and the sports industry not only promotes the transformation and upgrading of industrial business models but also promotes the transformation of industrial business operation logic and production relations. Although the combination of BT and the sports industry is still in its infancy, from the perspective of existing corporate practices, BT can help solve the core pain points of the sports industry ecosystem and will surely reshape the trust mechanism of the whole process and get through the sports industry chain with intellectual property as the core promotes the healthy operation, transformation, and upgrading of the ecosystem. The deep integration of the BT and the sports industry is not only a technical empowerment but also the practical application of decentralized concepts and the reconstruction of an integrated business model. With the advent of the $5 \mathrm{G}$ era, the sports industry and BT, big data, AI, AR/VR 1MR, and other technologies will be further integrated and expanded, which will inevitably bring new vitality to the sports industry, and promote the overall improvement of industrial operation efficiency. This not only requires the joint efforts of enterprises, governments, and society at the practical level but also requires crossborder and interdisciplinary research to provide groundbreaking and instructive academic support. 


\section{Data Availability}

No data were used to support this study.

\section{Conflicts of Interest}

The authors declared no potential conflicts of interest with respect to the research, authorship, and/or publication of this article.

\section{References}

[1] Y. Shan and Y. Mai, "Research on sports fitness management based on blockchain and internet of things," EURASIP Journal on Wireless Communications and Networking, vol. 2020, no. 1, Article ID 201, pp. 1-13, 2020.

[2] M. L. Naraine, "The blockchain phenomenon: conceptualizing decentralized networks and the value proposition to the sport industry," International Journal of Sport Communication, vol. 12, no. 3, pp. 313-335, 2019.

[3] X. Li, P. Jiang, T. Chen, X. Luo, and Q. Wen, "A survey on the security of blockchain systems," Future Generation Computer Systems, vol. 107, no. 1, pp. 841-853, 2020.

[4] F. Tian, "A supply chain traceability system for food safety based on HACCP, blockchain \& internet of things," in 2017 International Conference on Service Systems and Service Management, vol. 3 no. 4, pp. 585-598, Dalian, China, 2017.

[5] M. STOICA, B. GHILIC-MICU, M. MIRCEA, and P. SINIOROS, "E-government in a new technological ecosystem," Informatica Economica, vol. 24, no. 3/2020, pp. 5-15, 2020.

[6] O. Dong, "A study on the activating factors of the social creator industry," The Korean Journal of animation, vol. 12, no. 1, pp. 53-68, 2016.

[7] A. V. Konoplev, V. N. Golosov, V. I. Yoschenko et al., "Vertical distribution of radiocesium in soils of the area affected by the Fukushima Dai-ichi nuclear power plant accident," Eurasian Soil Science, vol. 49, no. 5, pp. 570-580, 2016.

[8] J. A. Wiens, "Methodologies for extrapolating from local to regional ecosystem scales: scaling functions and thresholds in animal responses to landscape pattern and land use," Orthopaedic Journal of Sports Medicine, vol. 2, no. 8, pp. 735-741, 2017.

[9] M. Stieler and C. C. Germelmann, "Actor engagement practices and triadic value co-creation in the team sports ecosystem," Marketing, vol. 40, no. 4, pp. 30-43, 2018.

[10] C. D. Luca and K. Hufeld, "An European ecosystem to boost the digitalization of EU industry Semi40 \& productive 4.0 ECSEL JU projects (2016-2020)," Ada user journal, vol. 38, no. 4, pp. 217-217, 2017.

[11] N. Marques, "The role of breakthrough technologies in the growth of esports," IEEE Potentials, vol. 38, no. 3, pp. 24-26, 2019.

[12] G. Lu, X. Zhou, and S. Li, "Statistical analysis-based Chinese sports industry listed companies competition factor and strategic performance," Journal of Computational and Theoretical Nanoscience, vol. 13, no. 12, pp. 10209-10213, 2016.

[13] L. Na, "Chinese sports industry listed companies' operation performance factor analysis," Journal of Computational and Theoretical Nanoscience, vol. 13, no. 12, pp. 9760-9764, 2016.
[14] A. Gvercn, "The factors that affect the development of sports industry as an economy," International journal of Science Culture and Sport, vol. 4, no. 18, pp. 515-515, 2016.

[15] S. Youn and H. Song, "Converging development between sports industry and elderly care service industry in China: connotations, status and trends," International Journal Of Human Movement Science, vol. 14, no. 1, pp. 43-59, 2020.

[16] R. A. Ziar, S. Irfanullah, W. U. Khan, and A. Salam, "Privacy preservation for on-chain data in the permission less blockchain using symmetric key encryption and smart contract," Mehran University Research Journal of Engineering and Technology, vol. 40, no. 2, pp. 305-313, 2021.

[17] S. Caneppele, F. Langlois, and P. Verschuuren, "Those who counter match-fixing fraudsters: voices from a multistakeholder ecosystem," Crime, Law and Social Change, vol. 74, no. 1, pp. 13-26, 2020.

[18] N. J. Zinia and P. Mcshane, "Urban ecosystems and ecosystem services in megacity Dhaka: mapping and inventory analysis," Urban Ecosystem, vol. 2021, no. 4, article 1076, pp. 1-14, 2021.

[19] T. Kharchenko and Z. Liu, "The relationship between sports industry development and economic growth in China," Accounting and Finance, vol. 1, no. 91, pp. 136-140, 2021.

[20] K. Bálint, "Possibilities for the utilization of an automatized, electronic blockchain-based, students' attendance register, using a universities' modern security cameras," Acta Polytechnica Hungarica, vol. 18, no. 2, pp. 127-142, 2021.

[21] Y. Gong, Q. Shi, D. Duan, and Z. Liu, "Research on application of intelligent tracing system for IVD reagents based on blockchain technology," Zhongguo yi liao qi xie za zhi = Chinese journal of medical instrumentation, vol. 45, no. 1, pp. 105108, 2021.

[22] Y. He, "A study on the impact of sport industry on economic growth: an investigation from China," Journal of Sports and Applied Science, vol. 2, no. 2, pp. 1-14, 2018.

[23] Y. Wang, M. Wang, J. Zhang, and B. Li, "Development trend of sports tourism industry based on econometric model from the perspective of macroeconomics," Boletin Tecnico/Technical Bulletin, vol. 55, no. 16, pp. 7-15, 2017.

[24] E. J. Seo and Y. S. Won, "A search for method development of silver sport industry through SWOT analysis," Korean Journal of Sports Science, vol. 28, no. 5, pp. 27-35, 2019. 